\title{
A NOVELTY APPROACH OF SPATIAL CO-OCCURRENCE AND DISCRETE SHEARLET TRANSFORM BASED TEXTURE CLASSIFICATION USING LPBOOSTING CLASSIFIER
}

\author{
${ }^{1}$ Vivek, C. and ${ }^{2}$ S. Audithan \\ ${ }^{1}$ PRIST University, Tanjore, Tamilnadu, India \\ ${ }^{2}$ Department of Computer Science and Engineering, PRIST University, Tamilnadu, India
}

Received 2013-12-18; Revised 2013-12-29; Accepted 2014-01-11

\begin{abstract}
Recently, the research towards Brodatz database for texture classification done at considerable amount of study has been published, the effective classification are vulnerable towards for training and test sets. This study presents the novel texture classification method based on feature descriptor, called spatial cooccurrence with discrete shearlet transformation through the LPboosting classification. It can be considered as a frame through the texton template that mapped into the texture images and it works directly on relating the adjacent spatial with its pixel boundary through the local intensity order. Hence, the proposed method for the feature extraction and classification of texture suggested with the experimentation through the spatial co-occurrence matrix with the power spectrum based discrete shearlet transform and it classified through the LP boosting method on Brodatz database images. This hybrid second order statistical based classification method significantly outperforms the existing texture descriptors the multiscale geometric tool shows the proposed method outperforms other classification method.
\end{abstract}

Keywords: Spatial Co-Occurrence Matrix, Texture Defect Detection, LPboost Classifier, Discrete Shearlet Transform, Texture Image Classification and Weak Classification, Autocorrelation, Power Spectrum

\section{INTRODUCTION}

With the increased research towards texture analysis on digital images and the corresponding computation based on various algorithms are key concepts for the sophisticated metrics to examine the features of the image. It is necessary to identifying the type of subject that varies for images due to illumination conditions. In this study, an image wherein there to identify the type of subject from black, white and grayscale image of photographs depicting the originality of the image based on the type of texture. The image features of various target images have been explained and features based on spatial Co-occurrence matrix are analyzed. In texture classification, the spatial based texture feature spaces are initially determined by Coggins and Jain (1985). The spatial gray tone co-occurrence probabilities with its spatial frequency in a texture based image are suggested by Hawkins (1970). The texton or texture perception based identical third-order statistics applied with the selective iso-trigon texture pair of black and white pixel square to discriminate the feature in the image studied by Julesz et al. (1978) The image texture primitives to the spatial dependence for the neighborhood pixels and their spatial distribution properties and pattern are derived for various types of data sets of aerial and satellite image by Haralick (1979). It suggested for microtextures, the statistical approach model such as gray tone co-occurrence evaluated widely. The spatial co-occurrence matrix method for grayscale image by selecting the optimal threshold values as an adaptive algorithm initially studied by Lie (1993).

Corresponding Author: Vivek, C., PRIST University, Tanjore, Tamilnadu, India 
The discrete Brodatz texture datasets are characterized through co-occurrence and it decomposed through wavelet transform that shows improves orthogonality but highly time consuming for due to multiscale strategy that provided by Unser (1995). The semivariogram is the extracted texture by using spatial autocorrelation function. The micro structure images as well as microwave remote-sensing images provide accurate results with the spatial co-occurrence matrices. This method is compared in the digital texture images provided by Carr and De Miranda (1998). The effective approach for feature comparison in Iris database to distinguish the various patterns of iris with reduced error rate as well as the fake identification in biometric applications delivered by Kumar et al. (2013). Later, the texture sea ice pattern of the Synthetic Aperture Radar (SAR) imagery are evaluated through Gray-Level CoOccurrence Matrix (GLCM) based on the entropy textural descriptors. It represents the image in a eightlevel Gaussian quantization and implemented through the Bayesian classifier for both microtextures and macrotextures image that provided by Soh and Tsatsoulis (1999). The Gabor Filters act as key role in Vehicle License Plate Detection (LPD) through the Neural Network classifiers applied by Ktata et al. (2013). The unsupervised Image template recognition is challenging task and the application of Optical Character Recognition (OCR) for Arabic handwritten numerals based on the dividing the numeral image the centroid point position and classifying it according to its shape of the horizontal projection by Zaghloul et al. (2012). The spatial metrics provide accurate information for the texture based image mapping in satellite imagery. The spatial structures are analyzed by the spatial heterogeneity of the land spaces and the Fisher Linear Discriminant (FLD) conducted for the land-use classification based on the examination of seven texture measurements and twenty-two spatial metrics. The accuracy level is 76.4 percent and it outperforms the other existing approach at that time that developed by Herold et al. (2003). The autocorrelation method evaluate the spatial distribution based on the primitives for the large MeasTex and VisTex datasets that classified through K-Nearest Neighbor classifier that shows significant performance that developed by Singh and Singh (2002). The medical based application for image retrieval through the feature extraction involves series of stages. The effective texture based analysis method named as Feature Optimized Classification Similarity (FOCS) frame work for diagnosis the medical image database developed by Jaganathan and Vennila (2013). Similarly, the Content Based Image Retrieval (CBIR) for clinical brain Magnetic Resonance Imaging (MRI) based on binary association rules that computed with trigonometric distance measure for accurately correlate between brain tumor tissues provided by Maheswari (2013). In our previous work focus primarily on the integrated the characterization of textures based on Discrete Shearlet Transform (DST) by extracting entropy measure and to classify the given Brodatz database texture image using K-Nearest Neighbor (KNN) classifier by Vivek and Audithan (2014). Although such adaptation improves the classification accuracy, it also severely increases the feature space complexity. Similarly, the an exclusive machine learning algorithm with strong supervised LPBoost classifier to train the ADNI database of MR images in a hyperplane shows improved in classification compared with other methods by Hinrichs et al. (2009). Similarly, the LPBoost algorithm optimized for weak classifier while ignoring strong classifier through minimax theory that revokes on the edge constraint shows higher convergence rate and accuracy for real world applications. Later, the same algorithm updated with strong classifier with the limited range that the training set of 5-fold-cross validation shows higher accuracy that studied by Fang et al. (2011). Thus, we propose the combination of multi-texton histogram with the Discrete Shearlet Transform (DST) to discriminate the Brodatz album based on feature extraction and then it undergoes classification with the minimax theory based LPboost classifier the accuracy of this system very well compared to other state of art techniques. In this proposed approach, the Brodatz database image feature are analyzed through the spatial co-occurrence of local intensity order and the extracted features are decomposed into various patterns by discrete shearlet transform and the obtained pattern are classified based on the similarity through the LP boosting method. The plant leaf classification based on the texture and homogeneity that compared with various classifiers enumerated by Ab Jabal et al. (2013). The rest of this study is organized as follows. In section II, the spatial co-occurrence of local intensity order are analyzed for the texture images are developed. In section III, the extracted feature images are represented through the DST to obtain the various decomposed patterns in the texture images. Then, the obtained pattern are classified based on the neighborhood similarity through the LP boosting method that presented in section IV, the 
experimental result are enumerated in section $\mathrm{V}$ and the study of conclusion in section VI.

\section{SPATIAL CO-OCCURRENCE MATRIX}

The texture features of the image are analyzed through the intensity based second order statistics feature descriptor named as co-occurrence matrices. It extracts the texture features based on the pixel pair probabilities. The adjacent correlated pixels are analyzed through the texton distinct templates. The texton is the series of pattern proposed by Liu et al. (2010). Qi et al. (2013) provided face recognition based on the local intensity order to enhance the descriptor based on the correlation between locally adjacent features and it shows significant recognition accuracy based on the developed patterns. It is the $2 \times 2$ grid of five unique combinations types of textons. It act as template for detecting the texture in the brodatz database images. Let $g$ be the unit vector corresponding to the $\mathrm{G}$ of the gray level in the image, then the following vectors co-ordinate with the function $\mathrm{f}(\mathrm{x}, \mathrm{y})$ Equation (1 and 2):

$\mathrm{u}=\frac{\partial \mathrm{G}}{\partial \mathrm{x}} \mathrm{g}$

$\mathrm{v}=\frac{\partial \mathrm{G}}{\partial \mathrm{y}} \mathrm{g}$

The dot products to the above vectors are given below Equation (3 to 5):

$$
\begin{aligned}
& g_{x x}=u^{T} u=\left|\frac{\partial G}{\partial x}\right|^{2} \\
& g_{y y}=v^{T} v=\left|\frac{\partial G}{\partial y}\right|^{2} \\
& g_{x y}=u^{T} v=\frac{\partial G}{\partial x} \bullet \frac{\partial G}{\partial y}
\end{aligned}
$$

The $\theta(\mathrm{x}, \mathrm{y})$ is the direction that changes with the vectors Equation (6):

$$
\theta(x, y)=\frac{1}{2} \tan ^{-1}\left[\frac{2 g_{x y}}{\left(g_{x x}-g_{y y}\right)}\right]
$$

To identify the value ranges $\mathrm{C}(\mathrm{x}, \mathrm{y})$ from lower value to higher value of 0 to 255 , the $\mathrm{G}(\mathrm{x}, \mathrm{y})$ is given below Equation (7):

$$
G(x, y)=\left\{\begin{array}{l}
\frac{1}{2}\left[\left(g_{x x}+g_{y y}\right)+\left(g_{x x}-g_{y y}\right)\right. \\
\left.\cos 2 \theta+2 g_{x y} \sin 2 \theta\right]
\end{array}\right\}^{\frac{1}{2}}
$$

To identify the texton in the original image, the texton templates are morphed the input image on various texton locations that generate the five unique combinations of texton component images. The texton templates consists of five types of unique frames to identify the textons that illustrated in Fig. 1.

According to the texton theory by Julesz et al. (1978), the image textures are decomposed based on the orientation, color variation and aspect ratios. The templates of similar feature in brodatz database image are mapped in the texture variation regions. Then, it is processed with the dictionary learning based methods. It generates the Local Intensity Order (LIO) that proposed by Wang et al. (2011). It describes the relationship between pixels in the four adjacent regions towards the mid-point. This LIO stimulate the cooccurrence form through it combines spatially. The spatial co-occurrence provides accurate information through the pair wise rotation invariant to the similar binary pattern. It helps to extract the feature of the image more robustly and the relative orders are stable to focus the relationship between the adjacent pixels. It also generates the locally adjacent patterns in the similar regions. It improves the discriminative power of the textural descriptors as it undergoes transformation. The pixel named " $A$ " has different pixel values in the boundary regions such $\mathrm{A}_{1}(62), \mathrm{A}_{2}(65), \mathrm{A}_{3}(58)$ and $\mathrm{A}_{4}(60)$. The region connects with other pixel named "B" through its one of the boundary. It helps to compare the local intensity order of the relative pixel regions as illustrated in Fig. 2.

The local binary patterns and the texton templates help to characterize the invariant pixel in the local regions. It generates the circular binary sequence since it convergence towards the center with its adjacent pixel regions. The developed LIO features $(\mathrm{F})$ are ordered in the four adjacent points as follows Equation (8):

$$
\mathrm{F}(\mathrm{P})=\operatorname{Order}\left(\mathrm{P}_{1}, \mathrm{P}_{2}, \mathrm{P}_{3}, \mathrm{P}_{4}\right)
$$



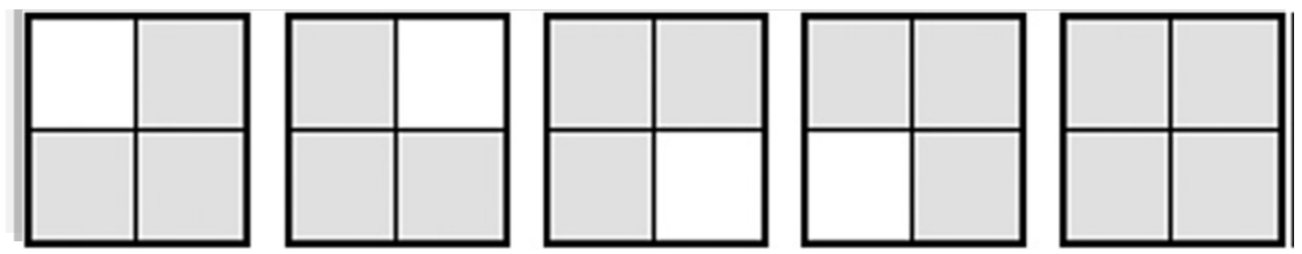

Fig. 1. Texton templates with five unique types

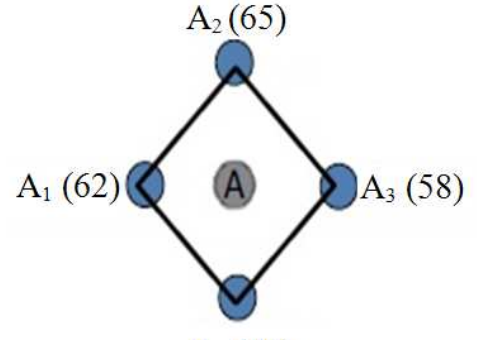

$\mathrm{A}_{4}(60)$

(a)

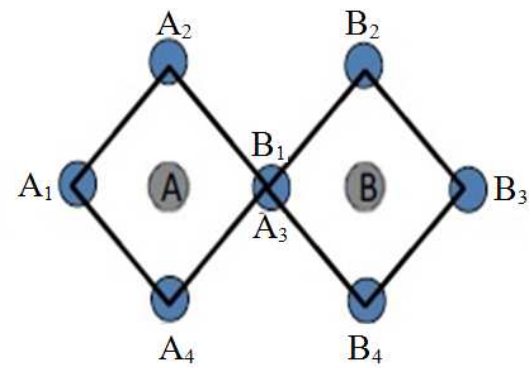

(b)

Fig. 2. (a) Pixel boundary of Local intensity order (LIO), (b) Adjacent LIO spatially related with another LIO

where, $\mathrm{P}_{1}, \mathrm{P}_{2}, \mathrm{P}_{3}, \mathrm{P}_{4}$ are the adjacent pixel points for the order function Order (.). Similarly, various LIO patterns are correlated in the spatially adjacent regions. It local features are arranged through the spatial co-occurrence matrix form to calculate the pixel regions after transformation of texture images. Thus, co-occurence of LIO that significantly boost the discriminative power of the similar feature is defined as Equation (9):

$$
F(P, Q)=\operatorname{Co}(f(P), f(Q))
$$

In Fig. 3, the broadatz image consists of numerous patterns. For a particular regions, the co-patterns such as $(4,5),(5,5)$ and $(23,24)$ for the certain positions mapping with the adjacent similar patterns. Then, the mapped two patterns are aligned into single pattern for effective corelation of the descriptors. The single pattern are the new labelled patterns and it compare with the another adjacent pixel regions to analyze the similarity of the pattern matching.

The LIO feature are extracted for the texture image and the co-occurence of LIO calculated based on the predefined texton template. It feature are enumerated in codebook size of 1024 to compare the other features. Then, block-wise representation are identified for the similar structural information. This representation are plotted in histogram and the concatenation feature are computed for the texture descriptor that go through the image transformation.

As shown in Fig. 4, the initial stage involves comparing the texton templates with the preprocessed image. It develops the sets of Local intensity order of many pixel regions pattern. It arranged into pixel-wise order for the effective analysis in the third stage. The arranged pixel pattern of the various Co-occurence of LIO (COLIO) are enumerated with the histogram. Finally, the histogram are compared to develop the descriptors.

\section{DISCRETE SHEARLET TRANSFORM}

The most significant step of any classification system is feature extraction. A new shearlet band signature, entropy is proposed based on discrete shearlet transform introduced by Glenn Easley. The group of $\mathrm{N}^{*} \mathrm{~N}$ database image that derived from the above texton sub space image. It consists of a finite sequence of values, $\left\{x\left[n_{1}, n_{2}\right]_{n_{1}, n_{2}=0}^{N-1, N-1}\right\}$ where $N \in \mathbb{N}$. Identifying the domain with the finite group $\mathbb{Z}_{\mathrm{N}}^{2}$, the inner product of image $\mathrm{x}, \mathrm{y}: \mathbb{Z}_{\mathrm{N}}^{2} \rightarrow \mathbb{C}$ is defined as Equation (10):

$$
(x, y)=\sum_{u=0}^{N-1} \sum_{v=0}^{N-1} x(u, v) \overline{y(u, v)}
$$


Vivek, C. and S. Audithan / Journal of Computer Science 10 (5): 783-793, 2014
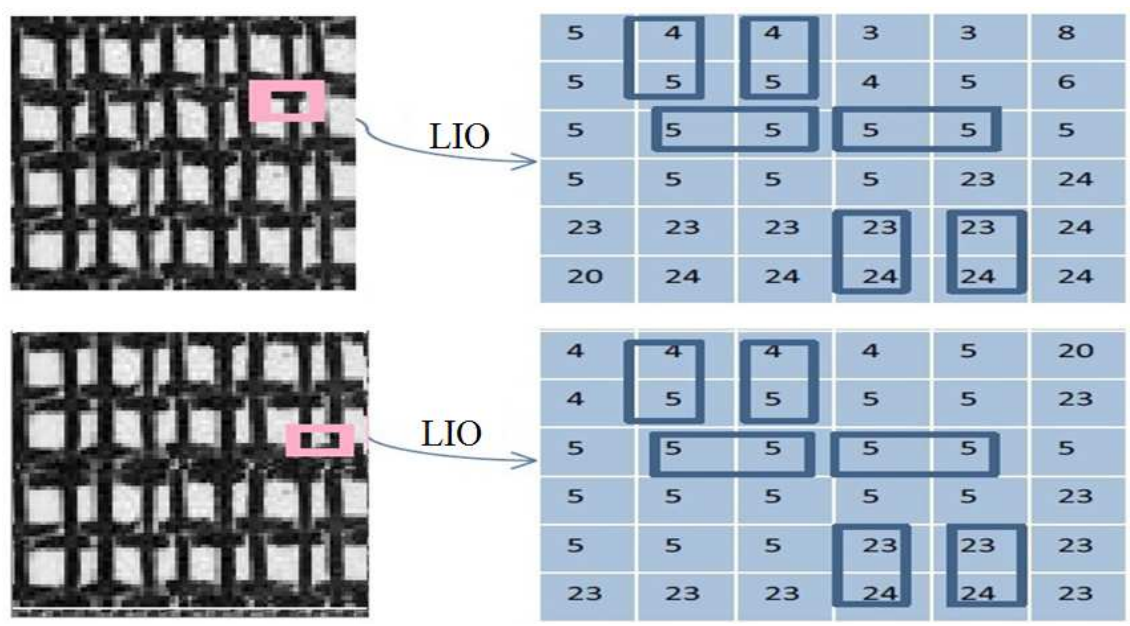

Fig. 3. LIO similarity pattern of Brodatz image

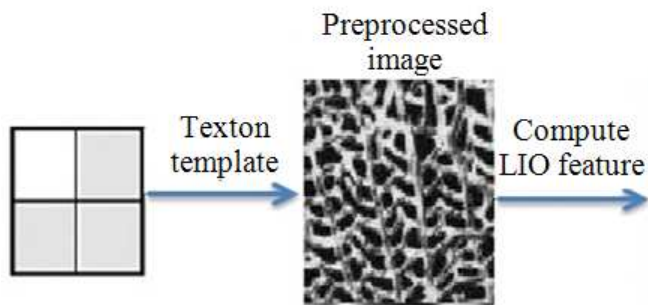

(b)

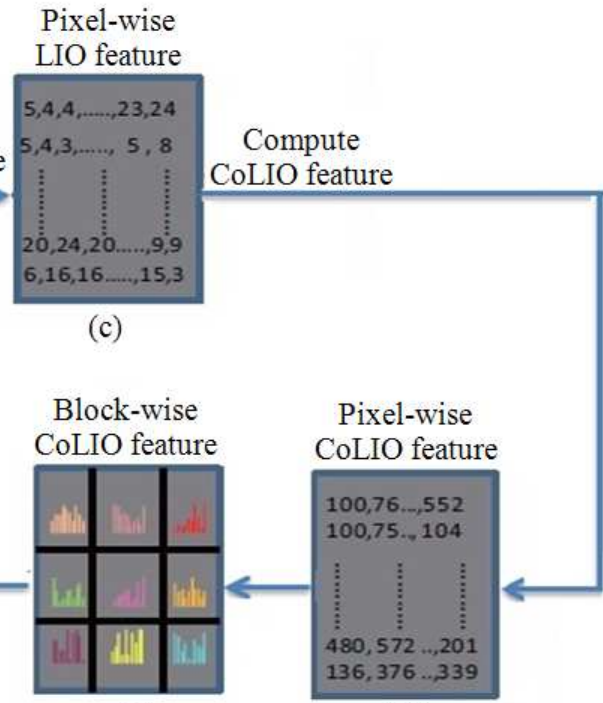

(e)

(d)

Fig. 4. The framework of the textural descriptor analysis through Co-occurerence of LIO

Thus the discrete analog of $\mathrm{L}^{2}\left(\mathbb{R}^{2}\right)$ is $1^{2} \mathbb{Z}_{\mathrm{N}}^{2}$. Given an image $\mathrm{f} \in \mathrm{l}^{2}\left(\mathbb{Z}_{\mathrm{N}}^{2}\right)$, let $\hat{\mathrm{f}}\left[\mathrm{k}_{1}, \mathrm{k}_{2}\right]$ denote its $2 \mathrm{D}$ Discrete Fourier Transform (DFT) Equation (11):

$$
\hat{\mathrm{f}}\left[\mathrm{k}_{1}, \mathrm{k}_{2}\right]=\frac{1}{\mathrm{~N}} \sum_{\mathrm{n}_{1}, \mathrm{n}_{2}=0}^{\mathrm{N}-1} \mathrm{f}\left[\mathrm{n}_{1}, \mathrm{n}_{2}\right] \mathrm{e}^{-2 \prod \mathrm{i}\left(\frac{\mathrm{n}_{1}}{\mathrm{~N}} \mathrm{k}_{1}+\frac{\mathrm{n}_{1}}{\mathrm{~N}} \mathrm{k}_{2}\right)}
$$

The brackets in the equations $[, \cdot]$ denote arrays of indices and parentheses $(\cdot, \cdot)$ denote function evaluations. Then the interpretation of the numbers $\hat{\mathrm{f}}\left[\mathrm{k}_{1}, \mathrm{k}_{2}\right]$ as samples $\hat{\mathrm{f}}\left[\mathrm{k}_{1}, \mathrm{k}_{2}\right]=\hat{\mathrm{f}}\left(\mathrm{k}_{1}, \mathrm{k}_{2}\right)$ is given by the following equation from the trigonometric polynomial Equation (12):

$$
\hat{\mathrm{f}}\left(\xi_{1}, \xi_{2}\right)=\sum_{\mathrm{n}_{1}, \mathrm{n}_{2}=0}^{\mathrm{N}-1} \mathrm{f}\left[\mathrm{n}_{1}, \mathrm{n}_{2}\right] \mathrm{e}^{-2 \prod \mathrm{i}\left(\frac{\mathrm{n}_{1}}{\mathrm{~N}} \xi_{1}+\frac{\mathrm{n}_{1}}{\mathrm{~N}} \xi_{2}\right)}
$$

First, to compute Equation (13): 


$$
\hat{\mathrm{f}}\left(\xi_{1}, \xi_{2}\right) \overline{\mathrm{V}\left(2^{-2 j} \xi_{1}, 2^{-2 j} \xi_{2}\right)}
$$

In the discrete domain, at the resolution level $\mathrm{j}$, the Laplacian pyramid algorithm is implemented in the time domain. This will accomplish the multi scale partition by decomposing $\mathrm{f}_{\mathrm{a}}^{\mathrm{j}-1}\left[\mathrm{n}_{1}, \mathrm{n}_{2}\right], 0 \leq \mathrm{n}_{1}, \mathrm{n}_{2}<\mathrm{N}_{\mathrm{j}}-1$ into a low pass filtered image $\mathrm{f}_{\mathrm{a}}^{\mathrm{j}}\left[\mathrm{n}_{1}, \mathrm{n}_{2}\right]$, a quarter of the size of $\mathrm{f}_{\mathrm{a}}^{\mathrm{j}-1}\left[\mathrm{n}_{1}, \mathrm{n}_{2}\right]$, and a high pass filtered image $\mathrm{f}_{\mathrm{d}}^{\mathrm{j}-1}\left[\mathrm{n}_{1}, \mathrm{n}_{2}\right]$. Observe that the matrix $\mathrm{f}_{\mathrm{a}}^{\mathrm{j}-1}\left[\mathrm{n}_{1}, \mathrm{n}_{2}\right]$ has size $\mathrm{N}_{\mathrm{j}} * \mathrm{~N}_{\mathrm{j}}$, where $\mathrm{N}_{\mathrm{j}}=2^{-2 \mathrm{j}} \mathrm{N}$ and $\mathrm{f}_{\mathrm{a}}^{0}\left[\mathrm{n}_{1}, \mathrm{n}_{2}\right]$ is equal to $\left[\mathrm{n}_{1}, \mathrm{n}_{2}\right]$ has size $N^{*} N$. In particular Equation (14):

$$
\hat{\mathrm{f}}_{\mathrm{d}}^{\mathrm{j}}\left(\xi_{1}, \xi_{2}\right)=\hat{\mathrm{f}}\left(\xi_{1}, \xi_{2}\right) \overline{\mathrm{V}\left(2^{-2 j} \xi_{1}, 2^{-2 j} \xi_{2}\right)}
$$

Thus, $\mathrm{f}_{\mathrm{d}}^{\mathrm{j}}\left[\mathrm{n}_{1}, \mathrm{n}_{2}\right]$ are the discrete samples of a function $\mathrm{f}_{\mathrm{d}}^{\mathrm{j}}\left[\mathrm{x}_{1}, \mathrm{x}_{2}\right]$ whose Fourier transform is $\hat{\mathrm{f}}_{\mathrm{d}}^{\mathrm{j}}\left(\xi_{1}, \xi_{2}\right)$. In order to obtain the directional localization the DFT on the pseudo-polar grid is computed and then one-dimensional band-pass filter is applied to the components of the signal with respect to this grid. More precisely, the definition of the pseudo-polar co ordinates $(\mathrm{u}, \mathrm{v}) \in \mathbb{R}^{2}$ as follows Equation (15 and 16):

$$
\begin{aligned}
& (\mathrm{u}, \mathrm{v})=\left(\xi_{1}, \frac{\xi_{2}}{\xi_{1}}\right), \text { if }\left(\xi_{1}, \xi_{2}\right) \in \mathrm{D}_{0} \\
& (\mathrm{u}, \mathrm{v})=\left(\xi_{1}, \frac{\xi_{1}}{\xi_{2}}\right), \text { if }\left(\xi_{1}, \xi_{2}\right) \in \mathrm{D}_{1}
\end{aligned}
$$

After performing this change of co ordinates, $\mathrm{g}_{\mathrm{j}}(\mathrm{u}, \mathrm{v})=\hat{\mathrm{f}}_{\mathrm{d}}^{\mathrm{j}}\left(\xi_{1}, \xi_{2}\right)$ is obtained and for $1=1-2^{\mathrm{j}}, \ldots 2^{\mathrm{j}}-1$ Equation (17):

$$
\begin{aligned}
\hat{\mathrm{f}}\left(\xi_{1}, \xi_{2}\right) & =\overline{\mathrm{V}\left(2^{-2 j} \xi_{1}, 2^{-2 \mathrm{j}} \xi_{2}\right) \mathrm{W}_{\mathrm{jl}}^{(\mathrm{d})}\left(\xi_{1}, \xi_{2}\right)} \\
& =\mathrm{g}_{\mathrm{j}}(\mathrm{u}, \mathrm{v}) \overline{\mathrm{W}\left(2^{\mathrm{j}} \mathrm{v}-1\right)}
\end{aligned}
$$

This expression shows that the different directional components are obtained by simply translating the window function $W$. The discrete samples $g_{j}\left[n_{1}, n_{2}\right]=$ $\mathrm{g}_{\mathrm{j}}\left(\mathrm{n}_{1}, \mathrm{n}_{2}\right)$ are the values of the DFT of $\mathrm{f}_{\mathrm{d}}^{\mathrm{j}}\left[\mathrm{n}_{1}, \mathrm{n}_{2}\right]$ on a pseudo-polar grid.

As shown in Fig. 5, the input dataset image evaluate into series of stages to classify based on the texture. The input image texture patterns are analyzed in the initial stage the spatial co-occurrence of local intensity order. It generates the descriptor based on the histogram analysis of the similar pattern of local intensity order of the adjacent pixels. Later, the descriptors are decomposed into various subbands through the second order entropy measure by the application of discrete shearlet transform. The logical views of the decomposition are exhibits in the following diagram. Finally, the decomposed images are classified for texture by the LP boosting classifier.

\section{STRONG LPBOOST CLASSIFIER}

The boosting classifier optimizes the classification based on edges " $\gamma$ " that applied in ADNI dataset by Hinrichs et al. (2009). The LPboost strong classifier focuses on the weak classifier for the extracted features based on the shearlet transform relative entropy. It bounds as the edges of the strong classifier in which " $\gamma$ " are lesser for minimum edges based on the convergence rate. The distributions of the edge margin are linear for training the set of images based on the similar features.

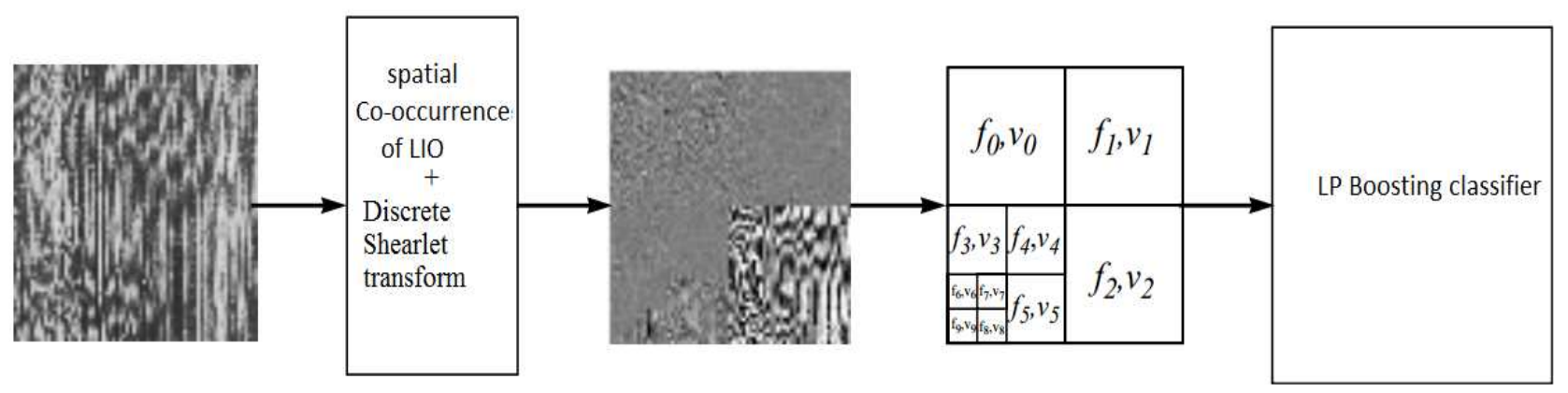

Fig. 5. The process of brodatz database image decomposition through TCM and DST 
The entropy regularized parameters for the feature vector, $\eta$ to update the distribution clearly. Based on the mini-max theory that eliminates the error in classifying though error matrix by Fang et al. (2010). In the Error matrix, the training sets $X=\left\{x_{1}, x_{2}\right.$, $\left.\mathrm{x}_{3} \ldots \mathrm{x}_{\mathrm{m}}\right\}$ and $\overline{\mathrm{d}}$ is the distribution of various training sets from $d_{1}, d_{2}, d_{3} \ldots d_{n}$ with the distribution of the hypothesis $\overline{\mathrm{w}}$ from $\mathrm{w}_{1}, \mathrm{w}_{2}, \mathrm{w}_{3} \ldots \mathrm{w}_{\mathrm{n}}$ that based on the features that manipulated with the hypothesis for each sample sets $h_{1}$, $\mathrm{h}_{2}, \mathrm{~h}_{3} \ldots \mathrm{h}_{\mathrm{n}}$. The minimax theory suggests the edge constraints $\sum_{\mathrm{i}=1}^{\mathrm{m}} \mathrm{H}_{\mathrm{t}-1}^{\prime}\left(\mathrm{x}_{\mathrm{i}}\right)$ based on its relative entropy through the feature extracted region. It helps to solve the weak classifier optimization effectively that developed by Fang et al. (2011). The main advantages of using LPboost classifiers are it performs train sequentially for the weak classifiers based on the preceding rules Equation (18):

$$
\begin{aligned}
& \mathrm{f}^{\mathrm{t}}(\mathrm{x})=\sum_{\mathrm{q}=1}^{\mathrm{t}} \mathrm{w}_{\mathrm{q}} \mathrm{h}^{\mathrm{q}}(\mathrm{x}) . \\
& \min _{\mathrm{d}_{\mathrm{t}}, \gamma} \gamma+\eta \cdot \Delta\left(\overline{\mathrm{d}}_{\mathrm{t}}, \overline{\mathrm{d}}_{0}\right) \\
& \text { s.t } \sum_{\mathrm{i}=1}^{\mathrm{m}} \mathrm{u}_{\mathrm{i}, \mathrm{j}} \mathrm{d}_{\mathrm{i}} \leq \gamma, \text { for } 1 \leq \mathrm{j} \leq \mathrm{t} ; \\
& \sum_{\mathrm{i}=1}^{\mathrm{m}} \mathrm{H}_{\mathrm{t}-1}^{\prime}\left(\mathrm{x}_{\mathrm{i}}\right) \mathrm{y}_{\mathrm{i}} \mathrm{d}_{\mathrm{i}} \leq \gamma ; \\
& 0 \leq \mathrm{d}_{\mathrm{j}} \leq \frac{\mathrm{v}}{\mathrm{m}}, \sum_{\mathrm{j}} \mathrm{d}_{\mathrm{j}}=1 ;
\end{aligned}
$$

It reduces the complication based on its hypotheses.

\section{EXPERIMENTAL RESULTS}

The performance evaluations of the proposed texture classification system are identified for the brodatz database images, $128 \times 128$ pixel sized images are extracted with an overlap of 32 pixels between vertical and horizontal direction. From a single 640x640 texture image, $256128 \times 128$ images are obtained. To carry out the proposed algorithm, 181 images are randomly selected from the 256 images. Among the 181 images, 101 images are used to test the classifier and the remaining 80 images are randomly chosen to train the classifier. It shows the 2 and 3-level shearlet transform used to decompose the texture images by varying the number of directions from 2 to 64 . Since the image are generated based on spatial co-occurrence of LIO with the DST is able to capture more information at higher directions. For 2level decomposition, the classification accuracy is 99.776\% (32 directions). The maximum classification accuracy achieved by 3-level decomposition with 64 directions is $99.866 \%$. The discriminate functions based on the LPboosting classification reduce the redundancy and misclassification. The classification rates abruptly increases as the training sets feature increases. It predominantly shows the efficient classification in the training images. Similarly, the collection of test images which identified by the subset homogenous pattern for classification. It improves the calculation and classification performance that shown in the below Fig. 6.

Table 1 and 2 shows the classification accuracy of the proposed texture classification system using shearlet entropy based on 2 and 3-level decomposition respectively. The numbers of directions in the 2-level DST for the texture images are evaluated in the ratio of $2,4,8,16,32,64$. It is noted that the classification accuracy increases from $91.45729 \%$ (2 directions) to 99.77554\% (32 directions) are shown in the Table 1. Similarly in Table 2, the classification rate for the 3level Discrete shearlet transform with the classification rate 97.2162 (2 directions) and it ultimately reaches maximum accurate texture classification of 99.8653 (64 directions).

\section{DISCUSSION}

In this method, the texture images in the brodatz database are selected. It consists of collection of unique texture images and there are many existing methods to analyze the texture features for this datasets. The existing methods include local features and kernels based texture analysis, sparse texture representation, fractal analysis, wavelet and GLCM, Linear Regression Model and the previous research method of DST based K-nearest neighbor classification that shows accuracy of 99.687. It is observed that the wavelet transform produces better performance than Gabor transform. Also the fusion of GLCM increases the classification accuracy. However, the proposed system based on DST 
outperforms all methods in terms of average classification accuracy. The comparative analysis of

the proposed method with other techniques is framed in the following Table 3.

Table 1. Classification rate of the proposed texture classification system using 2-level DST

\begin{tabular}{|c|c|c|c|c|c|c|}
\hline \multirow[b]{2}{*}{ Texture ID } & \multicolumn{6}{|c|}{ Number of directions } \\
\hline & 2 & 4 & 8 & 16 & 32 & 64 \\
\hline D6 & 93.23400 & 100.00000 & 100.00000 & 100.00000 & 100.00000 & 100.00000 \\
\hline D9 & 96.56700 & 100.00000 & 100.00000 & 100.00000 & 98.86100 & 100.00000 \\
\hline D11 & 93.94700 & 100.00000 & 100.00000 & 100.00000 & 100.00000 & 100.00000 \\
\hline D16 & 100.00000 & 100.00000 & 100.00000 & 100.00000 & 100.00000 & 100.00000 \\
\hline D17 & 96.32200 & 100.00000 & 100.00000 & 100.00000 & 100.00000 & 100.00000 \\
\hline D20 & 100.00000 & 100.00000 & 100.00000 & 100.00000 & 100.00000 & 100.00000 \\
\hline D21 & 100.00000 & 100.00000 & 100.00000 & 100.00000 & 100.00000 & 100.00000 \\
\hline D22 & 76.60980 & 100.00000 & 100.00000 & 100.00000 & 100.00000 & 100.00000 \\
\hline D24 & 93.68290 & 96.15200 & 100.00000 & 100.00000 & 100.00000 & 100.00000 \\
\hline D26 & 100.00000 & 100.00000 & 100.00000 & 100.00000 & 100.00000 & 100.00000 \\
\hline D34 & 98.84100 & 100.00000 & 100.00000 & 100.00000 & 100.00000 & 100.00000 \\
\hline D36 & 86.73900 & 98.86100 & 100.00000 & 97.82200 & 94.86290 & 88.53900 \\
\hline D41 & 96.50200 & 92.84390 & 100.00000 & 100.00000 & 100.00000 & 98.86100 \\
\hline D46 & 92.22900 & 100.00000 & 100.00000 & 100.00000 & 100.00000 & 100.00000 \\
\hline D47 & 93.68290 & 98.66100 & 100.00000 & 100.00000 & 100.00000 & 100.00000 \\
\hline D51 & 92.24390 & 96.42200 & 100.00000 & 100.00000 & 100.00000 & 100.00000 \\
\hline D53 & 100.00000 & 100.00000 & 100.00000 & 100.00000 & 100.00000 & 100.00000 \\
\hline D55 & 100.00000 & 100.00000 & 100.00000 & 100.00000 & 100.00000 & 100.00000 \\
\hline D56 & 100.00000 & 100.00000 & 100.00000 & 100.00000 & 100.00000 & 100.00000 \\
\hline D57 & 100.00000 & 100.00000 & 100.00000 & 100.00000 & 100.00000 & 100.00000 \\
\hline D64 & 100.00000 & 100.00000 & 100.00000 & 100.00000 & 100.00000 & 100.00000 \\
\hline D66 & 100.00000 & 100.00000 & 100.00000 & 100.00000 & 100.00000 & 100.00000 \\
\hline D68 & 100.00000 & 100.00000 & 100.00000 & 100.00000 & 100.00000 & 100.00000 \\
\hline D76 & 76.60980 & 93.88290 & 100.00000 & 100.00000 & 100.00000 & 100.00000 \\
\hline D77 & 100.00000 & 100.00000 & 100.00000 & 100.00000 & 100.00000 & 100.00000 \\
\hline D78 & 100.00000 & 100.00000 & 100.00000 & 100.00000 & 100.00000 & 100.00000 \\
\hline D79 & 91.24390 & 100.00000 & 100.00000 & 100.00000 & 100.00000 & 100.00000 \\
\hline D80 & 84.00800 & 81.73800 & 100.00000 & 100.00000 & 100.00000 & 100.00000 \\
\hline D82 & 100.00000 & 100.00000 & 100.00000 & 100.00000 & 100.00000 & 100.00000 \\
\hline D83 & 81.73800 & 100.00000 & 100.00000 & 100.00000 & 100.00000 & 100.00000 \\
\hline D85 & 50.18050 & 100.00000 & 100.00000 & 100.00000 & 100.00000 & 100.00000 \\
\hline D101 & 45.10240 & 61.73600 & 84.63800 & 79.95800 & 100.00000 & 59.85850 \\
\hline D102 & 79.64880 & 76.93800 & 89.43900 & 100.00000 & 98.16100 & 100.00000 \\
\hline D103 & 100.00000 & 100.00000 & 100.00000 & 100.00000 & 100.00000 & 100.00000 \\
\hline D104 & 100.00000 & 98.63100 & 100.00000 & 100.00000 & 100.00000 & 88.76590 \\
\hline D105 & 79.44880 & 86.45900 & 98.63100 & 100.00000 & 100.00000 & 36.46300 \\
\hline D106 & 69.79270 & 86.75900 & 97.25200 & 100.00000 & 99.36100 & 100.00000 \\
\hline D109 & 98.46100 & 100.00000 & 100.00000 & 100.00000 & 100.00000 & 100.00000 \\
\hline D111 & 100.00000 & 98.61300 & 100.00000 & 100.00000 & 100.00000 & 100.00000 \\
\hline Average & 91.45729 & 96.60761 & 99.22974 & 99.43026 & 99.77554 & 96.73045 \\
\hline
\end{tabular}


Vivek, C. and S. Audithan / Journal of Computer Science 10 (5): 783-793, 2014

Table 2. Classification rate of the proposed texture classification system using 3-level DST

\begin{tabular}{|c|c|c|c|c|c|c|}
\hline \multirow[b]{2}{*}{ Texture ID } & \multicolumn{6}{|c|}{ Number of directions } \\
\hline & 2 & 4 & 8 & 16 & 32 & 64 \\
\hline D6 & 100.0000 & 100.0000 & 100.0000 & 100.0000 & 100.0000 & 100.0000 \\
\hline D9 & 100.0000 & 100.0000 & 100.0000 & 100.0000 & 100.0000 & 100.0000 \\
\hline D11 & 99.1210 & 100.0000 & 100.0000 & 100.0000 & 100.0000 & 100.0000 \\
\hline D16 & 100.0000 & 100.0000 & 100.0000 & 100.0000 & 100.0000 & 100.0000 \\
\hline D17 & 100.0000 & 100.0000 & 100.0000 & 100.0000 & 100.0000 & 100.0000 \\
\hline D20 & 100.0000 & 100.0000 & 100.0000 & 100.0000 & 100.0000 & 100.0000 \\
\hline D21 & 100.0000 & 100.0000 & 100.0000 & 100.0000 & 100.0000 & 100.0000 \\
\hline D22 & 100.0000 & 100.0000 & 100.0000 & 100.0000 & 100.0000 & 100.0000 \\
\hline D24 & 100.0000 & 99.1210 & 100.0000 & 100.0000 & 100.0000 & 100.0000 \\
\hline D26 & 100.0000 & 100.0000 & 100.0000 & 100.0000 & 100.0000 & 100.0000 \\
\hline D34 & 100.0000 & 100.0000 & 100.0000 & 100.0000 & 100.0000 & 100.0000 \\
\hline D36 & 99.1210 & 100.0000 & 100.0000 & 100.0000 & 100.0000 & 100.0000 \\
\hline D41 & 100.0000 & 97.3820 & 100.0000 & 100.0000 & 100.0000 & 99.1210 \\
\hline D46 & 100.0000 & 100.0000 & 100.0000 & 100.0000 & 100.0000 & 100.0000 \\
\hline D47 & 100.0000 & 100.0000 & 100.0000 & 100.0000 & 100.0000 & 100.0000 \\
\hline D51 & 99.1210 & 100.0000 & 100.0000 & 100.0000 & 100.0000 & 100.0000 \\
\hline D53 & 100.0000 & 100.0000 & 100.0000 & 100.0000 & 100.0000 & 100.0000 \\
\hline D55 & 100.0000 & 100.0000 & 100.0000 & 100.0000 & 100.0000 & 100.0000 \\
\hline D56 & 100.0000 & 100.0000 & 100.0000 & 100.0000 & 100.0000 & 100.0000 \\
\hline D57 & 100.0000 & 100.0000 & 100.0000 & 100.0000 & 100.0000 & 100.0000 \\
\hline D64 & 100.0000 & 100.0000 & 100.0000 & 100.0000 & 100.0000 & 100.0000 \\
\hline D66 & 100.0000 & 100.0000 & 100.0000 & 100.0000 & 100.0000 & 100.0000 \\
\hline D68 & 100.0000 & 100.0000 & 100.0000 & 100.0000 & 100.0000 & 100.0000 \\
\hline D76 & 100.0000 & 100.0000 & 100.0000 & 100.0000 & 100.0000 & 100.0000 \\
\hline D77 & 100.0000 & 100.0000 & 100.0000 & 100.0000 & 100.0000 & 100.0000 \\
\hline D78 & 100.0000 & 100.0000 & 100.0000 & 100.0000 & 100.0000 & 100.0000 \\
\hline D79 & 100.0000 & 100.0000 & 100.0000 & 100.0000 & 100.0000 & 100.0000 \\
\hline D80 & 100.0000 & 100.0000 & 100.0000 & 100.0000 & 100.0000 & 100.0000 \\
\hline D82 & 100.0000 & 100.0000 & 100.0000 & 100.0000 & 100.0000 & 100.0000 \\
\hline D83 & 100.0000 & 100.0000 & 100.0000 & 100.0000 & 100.0000 & 100.0000 \\
\hline D85 & 97.3820 & 100.0000 & 100.0000 & 100.0000 & 100.0000 & 100.0000 \\
\hline D101 & 100.0000 & 100.0000 & 99.1210 & 100.0000 & 100.0000 & 100.0000 \\
\hline D102 & 100.0000 & 99.1210 & 99.1210 & 100.0000 & 99.1210 & 100.0000 \\
\hline D103 & 70.7370 & 68.2927 & 99.1210 & 97.3820 & 100.0000 & 99.1210 \\
\hline D104 & 78.0488 & 78.0488 & 97.3820 & 100.0000 & 100.0000 & 99.1210 \\
\hline D105 & 73.1707 & 97.3820 & 82.9268 & 91.3439 & 91.3439 & 97.3820 \\
\hline D106 & 75.6098 & 82.9268 & 99.1210 & 99.1210 & 100.0000 & 100.0000 \\
\hline D109 & 99.1210 & 99.1210 & 100.0000 & 100.0000 & 100.0000 & 100.0000 \\
\hline D111 & 100.0000 & 100.0000 & 100.0000 & 100.0000 & 100.0000 & 100.0000 \\
\hline Average & 97.2162 & 97.9845 & 99.4049 & 99.6884 & 99.7555 & 99.8653 \\
\hline
\end{tabular}

Table 3. Comparative analysis of the proposed system with other techniques

\begin{tabular}{|c|c|c|c|c|c|c|c|}
\hline Methods & $\begin{array}{l}\text { Wavelet and } \\
\text { GLCM }\end{array}$ & $\begin{array}{l}\text { (Van de Wouwer et al., } \\
\text { 1999) }\end{array}$ & $\begin{array}{l}\text { Linear Regression } \\
\text { Model }\end{array}$ & $\begin{array}{l}\text { (Wang and } \\
\text { Yong, 2008) }\end{array}$ & $\begin{array}{l}\text { DST based } \\
\text { KNN method }\end{array}$ & $\begin{array}{l}\text { (Vivek and } \\
\text { Audithan, 2014) }\end{array}$ & $\begin{array}{l}\text { Spatial Co- } \\
\text { occurrence and DST } \\
\text { based texture } \\
\text { classification using } \\
\text { LPboosting classifier }\end{array}$ \\
\hline Accuracy (\%) & 96.707 & & 97.151 & & 99.687 & & 99.852 \\
\hline
\end{tabular}




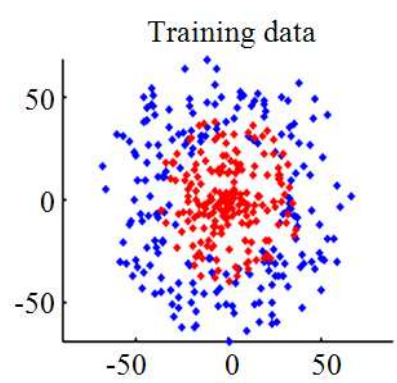

(a)

Test data classified with LPboost model

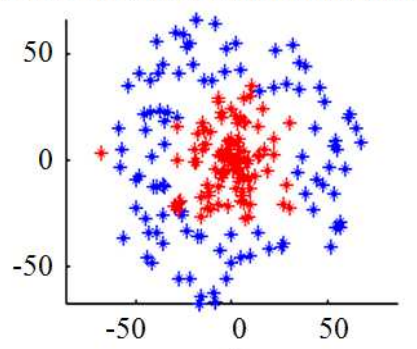

(b)

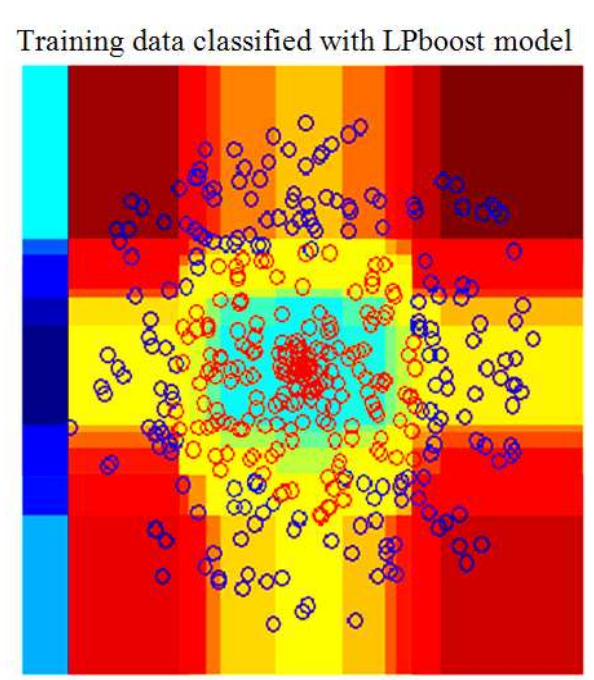

(c)

Fig. 6. LPboosting classification on the texture images: (a) Training data, (b) Test data classified with LPboost model, (c) Training data classified with LPboost model

\section{CONCLUSION}

In this study, the texture classification was performed using the combination of text on template with the spatial based co-occurrence of local intensity order and it generates the texture descriptor that decomposed into transformation pattern by the shearlet band signatures based texture classification for the database of 181 Brodatz texture images is investigated. The feature extraction done more effectively and the sub-band are analyzed through the entropy measures and it undergoes for classification through LPboosting which provokes on weak classifier as the training images and then used to classify the testing images. The proposed system consistently achieves over $99.865 \%$ classification accuracy. Experimental results show that the proposed spatial Co-occurrence of LIO based DST outperforms various existing wavelet and Gabor transform based classification systems. In future work, we will extend this proposed hybrid method of spatial co-occurrence with discrete shearlet transform method for feature extraction in the medical images. The medical image such as MRI, CT and Ultrasound requires sophisticated methodology for extract the feature in the image to classify the type of regions. It is very important evaluation work to segment the affected tissue regions.

\section{REFERENCES}

Ab Jabal, M.F., S. Hamid, S. Shuib and I. Ahmad, 2013. Leaf features extraction and recognition approaches to classify plant. J. Comput. Sci., 9: 1295. DOI: 10.3844/jcssp.2013.1295.1304

Carr, J.R. and F.P. De Miranda, 1998. The semivariogram in comparison to the co-occurrence matrix for classification of image texture. IEEE Trans. Geosci. Remote Sens., 36: 1945-1952. DOI: 10.1109/36.729366

Coggins, J.M. and A.K. Jain, 1985. A spatial filtering approach to texture analysis. Patt. Recogn. Lett., 3: 195-203. DOI: 10.1016/0167-8655(85)90053-4

Fang, Y., Y. Fu, C. Sun and J. Zhou, 2011. Improved boosting algorithm using combined weak classifiers. J. Computat. Inform. Syst., 7: 1455-1462.

Fang, Y.K., Y. Fu, C.J. Sun and J.L. Zhou, 2010. LPBoost with strong classifiers. Int. J. Computat. Intell. Syst., 3: 88-100. DOI: 10.1080/18756891.2010.9727755 
Haralick, R.M., 1979. Statistical and structural approaches to texture. Proc. IEEE, 67: 786-804. DOI: 10.1109/PROC.1979.11328

Hawkins, J.K., 1970. Textural properties for pattern recognition. Picture Process. Psychopictor.

Herold, M., X. Liu and K.C. Clarke, 2003. Spatial metrics and image texture for mapping urban land use. Am. Soc. Photogrammetric Eng. Remote Sens., 69: 991-1002. DOI: 10.14358/PERS.69.9.991

Hinrichs, C., V. Singh, L. Mukherjee, G. Xu and M.K. Chung et al., 2009. Spatially augmented LPboosting for $\mathrm{AD}$ classification with evaluations on the ADNI dataset. Neuroimage, 48: 138-149. DOI: 10.1016/j.neuroimage.2009.05.056

Jaganathan, Y. and I. Vennila, 2013. A hybrid approach based medical image retrieval system using feature optimized classification similarity framework. Am. J. Applied Sci., 10: 549-562. DOI: 10.3844/ajassp.2013.549.562

Julesz, B., E.N. Gilbert and J.D. Victor, 1978. Visual discrimination of textures with identical third-order statistics. Biol. Cybernet., 31: 137-140. DOI: 10.1007/BF00336998

Ktata, S., F. Benzarti and H. Amiri, 2013. License plate localization using Gabor filters and neural networks. J. Comput. Sci., 9: 1341-1347. DOI: 10.3844/jcssp.2013.1341.1347

Kumar, M.R., M.D. Fathima, K. Kiruthika and M.S. Saravanan, 2013. Non-cooperative iris recognition: A novel approach for segmentation and fake identification. J. Comput. Sci., 9: 1241-1251. DOI: $10.3844 /$ jcssp.2013.1241.1251.

Lie, W.N., 1993. An efficient threshold-evaluation algorithm for image segmentation based on spatial gray level co-occurrences. Signal Process., 33: 121126. DOI: 10.1016/0165-1684(93)90083-M

Liu, G.H., L. Zhang, Y.K. Hou, Z.Y. Li and J.Y. Yang, 2010. Image retrieval based on multi-texton histogram. Patt. Recogn., 43: 2380-2389. DOI: 10.1016/j.patcog.2010.02.012

Maheswari, U., 2013. Content based medical image retrieval using binary association rules. J. Comput. Sci., 9: 678-689. DOI: 10.3844/jcssp.2013.678.689

Qi, X., Y. Lu, S. Chen, C.G. Li and J. Guo, 2013. Spatial co-occurrence of local intensity order for face recognition. Proceedings of the IEEE International Conference on Multimedia and Expo Workshops, Jul. 15-19, IEEE Xplore Press, San Jose, CA., pp: 16. DOI: 10.1109/ICMEW.2013.6618447
Singh, M. and S. Singh, 2002. Spatial texture analysis: A comparative study. Proceedings of the 16th International Conference on Pattern Recognition, Aug. 11-15, IEEE Xplore Press, pp: 676-679. DOI: 10.1109/ICPR.2002.1044843

Soh, L.K. and C. Tsatsoulis, 1999. Texture analysis of SAR sea ice imagery using gray level co-occurrence matrices. IEEE Trans. Geosci. Remote Sens., 37: 780-795. DOI: $10.1109 / 36.752194$

Unser, M., 1995. Texture classification and segmentation using wavelet frames. IEEE Trans. Image Process., 4: 1549-1560. DOI: 10.1109/83.469936

Van de Wouwer, G., P. Scheunders and D. Van Dyck, 1999. Statistical texture characterization from discrete wavelet representations. IEEE Trans. Image Process., 8: 592-598. DOI: 10.1109/83.753747

Vivek, C. and S. Audithan, 2014. Texture classification by shearlet Band Signatures. Asian J. Scient. Res.

Wang, Z., B. Fan and F. Wu, 2011. Local intensity order pattern for feature description. Proceedings of the IEEE International Conference on In Computer Vision, Nov. 6-13, IEEE Xplore Press, Barcelona, pp: 603-610. DOI: 10.1109/ICCV.2011.6126294

Wang, Z.Z. and J.H. Yong, 2008. Texture analysis and classification with linear regression model based on wavelet transform. IEEE Trans. Image Process., 17 : 1421-1430. DOI: 10.1109/TIP.2008.926150

Zaghloul, R.I., D.M.B. Enas and F. AlRawashdeh, 2012. Recognition of Hindi (Arabic) handwritten numerals. Am. J. Eng. Applied Sci., 5: 132-135. DOI: 10.3844/ajeassp.2012.132.135 